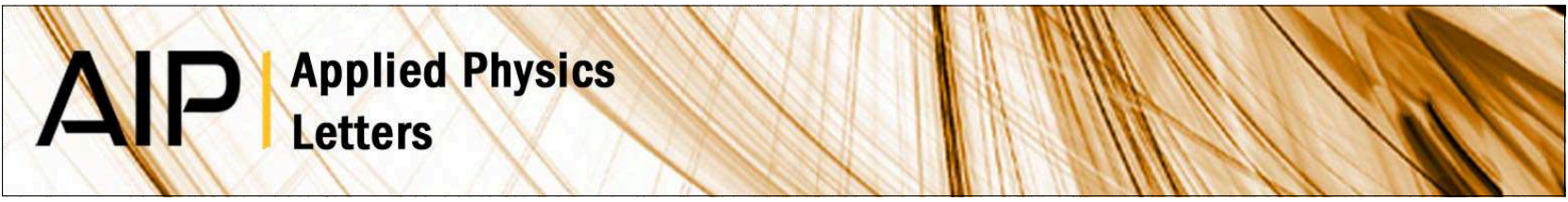

\section{Modeling and performance of vanadium-oxide transition edge microbolometers}

L. A. L. de Almeida, G. S. Deep, A. M. N. Lima, I. A. Khrebtov, V. G. Malyarov et al.

Citation: Appl. Phys. Lett. 85, 3605 (2004); doi: 10.1063/1.1808890

View online: http://dx.doi.org/10.1063/1.1808890

View Table of Contents: http://apl.aip.org/resource/1/APPLAB/v85/i16

Published by the American Institute of Physics.

\section{Related Articles}

Infrared detectors based on semiconductor $\mathrm{p}-\mathrm{n}$ junction of $\mathrm{PbSe}$

J. Appl. Phys. 112, 086101 (2012)

A titanium-nitride near-infrared kinetic inductance photon-counting detector and its anomalous electrodynamics Appl. Phys. Lett. 101, 142602 (2012)

GeSn p-i-n detectors integrated on Si with up to $4 \% \mathrm{Sn}$

Appl. Phys. Lett. 101, 141110 (2012)

Energy free microwave based signal communication using ratchet effect

Appl. Phys. Lett. 101, 143504 (2012)

Superconducting detector dynamics studied by quantum pump-probe spectroscopy

Appl. Phys. Lett. 101, 112603 (2012)

\section{Additional information on Appl. Phys. Lett.}

Journal Homepage: http://apl.aip.org/

Journal Information: http://apl.aip.org/about/about_the_journal

Top downloads: http://apl.aip.org/features/most_downloaded

Information for Authors: http://apl.aip.org/authors

\section{ADVERTISEMENT}
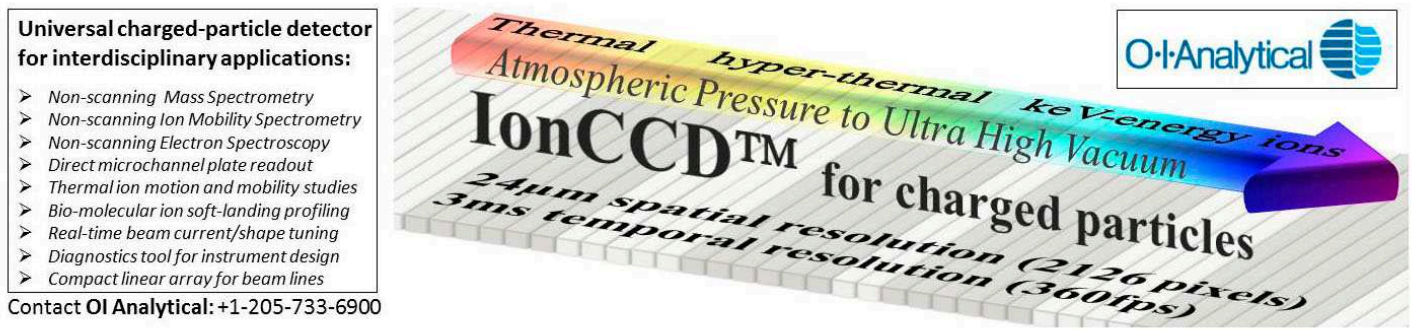


\title{
Modeling and performance of vanadium-oxide transition edge microbolometers
}

\author{
L. A. L. de Almeida \\ Department of Electrical Engineering, Universidade Federal da Bahia R. Aristides Novis no. 2, \\ 40210-630 Salvador, BA, Brazil \\ G. S. Deep and A. M. N. Lima ${ }^{\text {a) }}$ \\ Department of Electrical Engineering, Universidade Federal de Campina Grande, \\ Av. Aprigio Veloso no. 882, 58109-970 Campina Grande, PB, Brazil \\ I. A. Khrebtov and V. G. Malyarov \\ Vavilov State Optical Institute, 12, Birzhevaya line, St. Petersburg 199034, Russia \\ H. Neff \\ VIR A/S, Kuldyssen 10, 2630 Taastrup, Denmark
}

(Received 11 November 2003; accepted 24 August 2004)

\begin{abstract}
The performance of a $\mathrm{VO}_{2}$ thin-film microbolometer has been investigated. The device is operated within $35^{\circ} \mathrm{C}<T<60^{\circ} \mathrm{C}$, in the hysteretic metal-insulator transition region. An algebraic hysteresis model has been used to model the resistance-temperature characteristic of the sensor. It accurately describes the resistance versus temperature characteristics of the material. Employing this model, and in conjunction with established bolometer theory, the responsivity of a $\mathrm{VO}_{2}$ film is calculated and compared with experimental data. Superior performance of the device is achievable under conditions of single pulse incident radiation where the operating point remains on the major hysteresis loop. This results in a pronounced responsivity peak within the center of the metal-insulator transition. Continuous periodic excitation, in contrast, leads to a steadily decreasing and much lower sensitivity at higher temperature, due to the formation of minor hysteresis loops and the loop accommodation process. (C) 2004 American Institute of Physics. [DOI: 10.1063/1.1808890]
\end{abstract}

Bolometric detectors for infrared and millimeter electromagnetic radiation are of considerable scientific and technical interest for spectroscopy, communications, and remote sensing. ${ }^{1,2}$ These detectors employ an electrical resistance thermometer to measure temperature variations, caused by incident radiation. The bolometer sensitivity is directly related to its temperature coefficient of resistance (TCR). It depends on both the type of material and operating temperature of the thermometer. Cooled bolometers employ very sensitive thermometers, and operate at or below liquid nitrogen temperature. For many applications, this low temperature requirement is a serious drawback, especially in the design of microbolometer arrays for portable infrared sensing. Vanadium dioxide $\left(\mathrm{VO}_{2}\right)$ is a commonly employed material for fabrication of microbolometer detector arrays.

Thin $\mathrm{VO}_{2}$ films exhibit a temperature driven solid state phase transition slightly above ambient temperature. Over a narrow temperature range of a few degrees, the electrical resistance of thin films may change several orders of magnitude. This accounts for large negative temperature coefficients of resistance, approaching $200 \% / \mathrm{K}$ for high quality films. ${ }^{3}$ Despite these large resistance temperature coefficients, present uncooled bolometers, based on heat sensing $\mathrm{VO}_{2}$ films are operated in the nonhysteretic semiconducting part of its resistance-temperature $(R-T)$ curve, at temperatures below the metal-insulator $(\mathrm{M}-\mathrm{I})$ transition. ${ }^{4}$

Recently, an experimental study of the responsivity of a $\mathrm{VO}_{2}$ bolometer operating in the hysteretic region has been

\footnotetext{
a) Author to whom correspondence should be addressed; electronic mail: amnlima@dee.ufcg.edu.br
}

reported. ${ }^{5}$ In terms of bolometric responsivity, the large resistance temperature coefficients of $\mathrm{VO}_{2}$ films should result in superior device performance. However, the metalinsulator transition of $\mathrm{VO}_{2}$ thin films is hysteretic in nature, and the mechanism of operation in the transition region becomes rather complex, and is not yet fully understood. ${ }^{6}$ Here, we present the performance analysis of the $\mathrm{VO}_{2}$ transition edge bolometer based on the algebraic model reported in Refs. 7 and 8 and the results are compared with the experimental results reported in Ref. 5.

Bolometric performance analysis has been done, combining the bolometric heat balance equation with an appropriately adapted hysteresis model for the sensor resistance $(R-T){ }^{7}$ The thermoresistive element, biased with electrical power $I^{2} R(T)$, the bolometric heat balance equation in constant current mode (CCM) can be written as

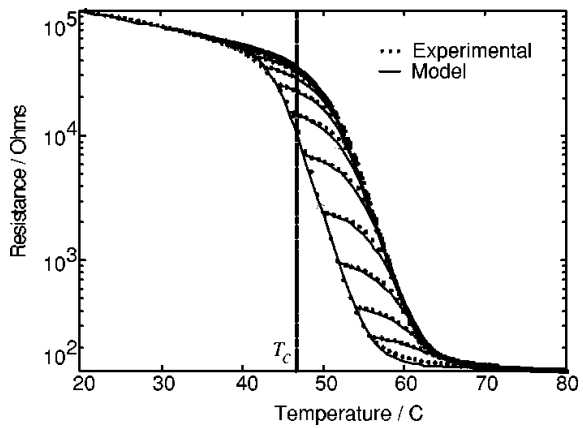

FIG. 1. Experimentally measured first-order $R \times T$ descending curves for the film resistance (solid circles) and those calculated from the model (2) and (6) (solid line). 


$$
C \frac{d T}{d t}=\eta P+I^{2} R(T)-G\left(T-T_{h}\right),
$$

where $P$ is the incident radiation power, $\eta$ is the absorbance of the bolometer, $I$ is the constant bias current, $G$ is the thermal conductivity to the heat sink, $C$ is the heat capacity of the sensitive element, $T_{h}$ is the heat sink temperature (assumed to remain constant) and $R(T)$ is the temperature dependent sensor resistance. In the CCM with an NTC sensor the operation is inherently stable, providing the shortest response time. Details regarding bias dependent dynamics of bolometer operation are presented elsewhere.

The hysteretic temperature induced resistance variation $R(T)$ of a $\mathrm{VO}_{2^{-}}$film, having a negative TCR, can be written as

$$
R(T)=17 \exp \left(\frac{2553}{T+273}\right) g(T)+140,
$$

where $g(T)$ denotes the semiconducting volume fraction at any point inside the major limiting hysteresis loop and is given by

$$
g(T)=\frac{1}{2}+\frac{1}{2} \tanh \beta\left[\delta \frac{w}{2}+T_{c}-\left(T+T_{\mathrm{pr}} P\left(\frac{T-T_{r}}{T_{\mathrm{pr}}}\right)\right)\right]
$$

where $w$ is the hysteresis width, $T_{c}$ is the critical temperature or percolation threshold, $\beta$ is related to $d g / d T$ at $T_{c}$, and $\delta$ is an operator defined as

$$
\delta=\operatorname{sign}(d T / d t)
$$

$T_{\mathrm{pr}}$ is the proximity temperature at the start of a new branch and from Eq. (3) can be written as

$$
T_{\mathrm{pr}}=\delta \frac{w}{2}+T_{c}-\frac{1}{\beta} \operatorname{arctanh}\left(2 g_{r}-1\right)-T_{r} .
$$

The coordinates of the reversal point are denoted by $\left(T_{r}, g_{r}\right)$ and $P(x)$, for $x=T-T_{r} / T_{\mathrm{pr}}$, is an arbitrary monotonically decreasing function, with $P(0)=1$, called the proximity function, and given, for the film employed in this work, as

$$
P(x) \triangleq \frac{1}{2}[1-\sin (\gamma x)]\left[1+\tanh \left(\pi^{2}-2 \pi x\right)\right],
$$

where $\gamma$ is an arbitrary constant.

The numerical solution of $\mathrm{VO}_{2}$ thin film hysteresis model described by Eqs. (2)-(6) is relatively simple to obtain. Further details, regarding the derivation, numerical approach, accuracy, performance and application of the presently employed general hysteresis model are presented elsewhere. 7,8

Details regarding the fabrication and characteristics of the films employed in this work are given in Ref. 5. A digital temperature controller, implemented in a computer supervised environment, was employed to obtain a high precision temperature ramping and measurement of the film resistance. ${ }^{10}$ The values obtained for the parameters are: ${ }^{7} w$ $=6.5 \mathrm{C}, T_{c}=47.6^{\circ} \mathrm{C}, \beta=0.2{ }^{\circ} \mathrm{C}^{-1}$ and $\gamma=0.85$. In Fig. 1 , a set of experimentally obtained first-order descending $R \times T$ curves (solid circles) is illustrated.

The output voltage of the bolometer operated in CCM and neglecting electrothermal feedback effects, with incident radiation at frequency $f$, can be written as ${ }^{11}$

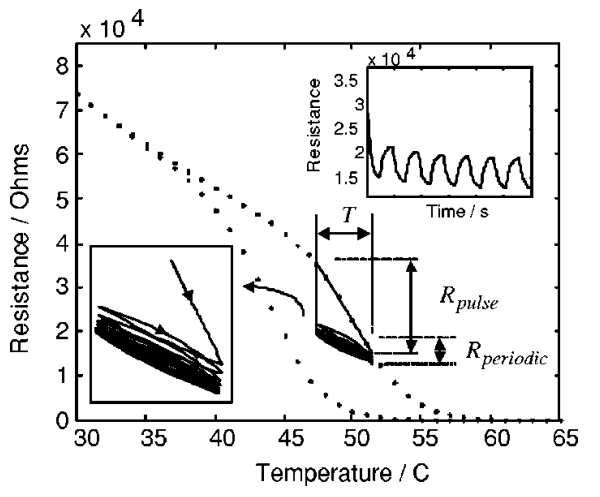

FIG. 2. Simulation results for a pulsed periodic incident radiation $\eta P$ which produces a temperature variation $\Delta T$. In the right hand inset is shown the larger resistance variation $\Delta R_{\text {pulse }}$ for the first pulse of the incident radiation. This effect can also be observed in the $R \times T$ characteristic. After the first pulse, due to the hysteretic behavior, the minor loop drifts downwards and after a few cycles, it becomes stable with resistance variations assuming a smaller value $\Delta R_{\text {periodic }}$.

$$
S(f)=\eta I \frac{d R(T)}{d T}|G+j 2 \pi f C|^{-1}[\mathrm{~V} / \mathrm{W}]
$$

where $G=1.9 \times 10^{-4} \mathrm{~W} / \mathrm{K}$ and $C=2.85 \times 10^{-5} \mathrm{~J} / \mathrm{K}$ were experimentally determined. ${ }^{5}$ The sensor bias current is set to $I=15.0 \mu \mathrm{A}$.

The full set of Eqs. (1), (2), and (6) can be used to represent the nonlinear dynamics of the bolometer. The initial conditions for simulation are set to allow the $\mathrm{VO}_{2}$ bolometer to operate on the major limiting descending curve for several operating temperatures over the range between 20 and $80^{\circ} \mathrm{C}$. The operating temperature is established by setting the heat sink temperature $T_{h}$ to a certain value as a control parameter. The initial film temperature is set at $T_{0}$ $=15{ }^{\circ} \mathrm{C}$ which accounts for the simulation starting point to be always with $d T / d t>0$ for any heat sink temperature $T_{h}$ between 20 and $80^{\circ} \mathrm{C}$.

Figure 2 shows the bolometer temporal response (upper right side inset) to repetitive incident radiation pulses, that has been maintained in thermal equilibrium before applying the periodic pulse incident radiation, i.e., $T \approx T_{h}$. The resistance variation to the first pulse of the periodic incident radiation is denoted $\Delta R_{\text {pulse }}$, whereas the resistance variation due to the following pulses is denoted by $\Delta R_{\text {periodic }}$, as it is shown in the $R \times T$ characteristic of Fig. 2. After the first pulse, due to the hysteretic behavior, radiation induced relatively small temperature variations provoke minor loop formation. These minor loops drift slowly downwards, comprising substantially smaller TCR values. After a few cycles, stabilization occurs for the resistance variations $\Delta R_{\text {periodic }}$; this is the result of so-called minor loop accommodation process. $^{7}$

The normalized voltage responsivities were calculated from simulation results for each operating temperature $T$ $\approx T_{h}$ as

$$
\begin{aligned}
& S_{\text {pulse }}\left(T_{h}\right)=\frac{\Delta R_{\text {pulse }} / \Delta T}{\Delta R_{\text {pulse }} /\left.\Delta T\right|_{T_{h}=20{ }^{\circ} \mathrm{C}}}, \\
& S_{\text {periodic }}\left(T_{h}\right)=\frac{\Delta R_{\text {periodic }} / \Delta T}{\Delta R_{\text {periodic }} /\left.\Delta T\right|_{T_{h}=20{ }^{\circ} \mathrm{C}}} .
\end{aligned}
$$




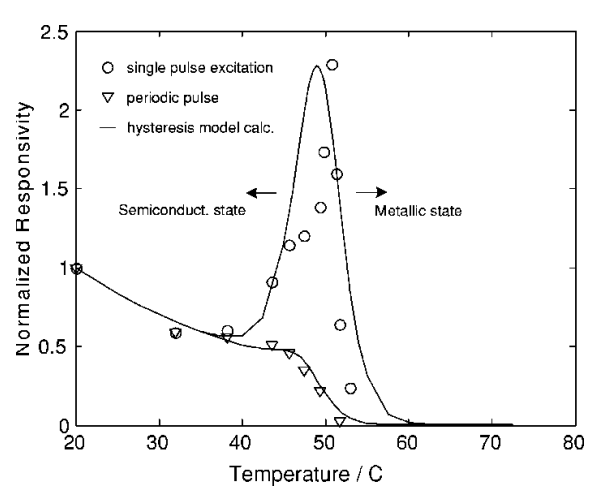

FIG. 3. The experimentally obtained normalized voltage responsivities for single pulse (circles) and periodic (triangles) incident radiations, as well as those (solid lines) calculated from the model (1), (2) and (6) by means of (8) and (9), respectively.

The normalized voltage responsivities were calculated from the calculated values of the sensor resistance variations resulting from the incident radiation.

In Fig. 3 experimental and calculated normalized output voltage responsivities are presented. For single pulse excitation a pronounced maximum occurs at $T=50^{\circ} \mathrm{C}$, near the center of the M-I transition. Its magnitude is approximately a factor of 2.5 higher than the value observed in the fully semiconducting nonhysteretic region below $20^{\circ} \mathrm{C}$. In contrast, for periodic excitation a continuous decrease of the responsivity at higher $T$ is observable. At $50{ }^{\circ} \mathrm{C}$ the responsivity for periodic pulsed incident radiation is about a factor of 12 lower than that for single pulse radiation. Using special ac biasing technique to operate the bolometer in the hysteretic phase transition region, an improvement in responsivity by a factor of 5 as compared to that obtained with a constant current bias was reported in Ref. 3. The difference between calculated and measured responsivities for single pulse incident radiation near $50{ }^{\circ} \mathrm{C}$ (see Fig. 3) is yet not fully clear to us. It is likely that the thermosensitive film was not monophase and contained some amount of other vanadium oxides besides $\mathrm{VO}_{2}$.

The relevant figure of merit of bolometer operation is the noise equivalent power (NEP) defined as

$$
\mathrm{NEP}=\frac{V_{n}(f)}{S(f)}\left[\mathrm{W} / \mathrm{Hz}^{1 / 2}\right],
$$

where $V_{n}(f)$ is the noise voltage for $1 \mathrm{~Hz}$ bandwidth and $S(f)$ is the frequency dependent responsivity in the hysteretic transition region. Presently, there is only limited information available regarding the noise characteristics of $\mathrm{VO}_{2}$ films. It has been suggested that flicker, or $1 / f$ noise, is the dominating noise source ${ }^{3,12,13}$ and its origin assigned to the martensitic transformations occurring within the film.

At present it is not possible to develop a more complete model for estimation of NEP value. Nevertless, the gain in the NEP can be estimated using experimental data of the bolometer noise measurements. In Fig. 4 the experimentally obtained bolometer noise spectra for operating temperatures of 18 and $50{ }^{\circ} \mathrm{C}$ with constant current bias of $15 \mu \mathrm{A}$ are presented (the experimental results shown in Fig. 3 were also obtained for the same bias current). At the frequency of $1 \mathrm{~Hz}$, at which the bolometer responsivity exhibits a considerably reduced dependence on frequency, the noise voltage at

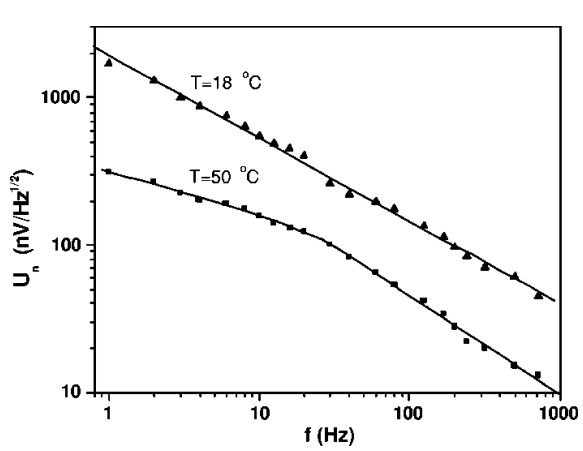

FIG. 4. Experimental frequency dependence of the bolometer noise voltage at operating temperatures 18 and $50^{\circ} \mathrm{C}$ with constant current bias of $15 \mu \mathrm{A}$.

$50{ }^{\circ} \mathrm{C}$ is a factor of 6 less than that at $18^{\circ} \mathrm{C}$. This is due to the differences in noise magnitudes and in the slopes of the two curves shown in Fig. 4 over the frequency range below $30 \mathrm{~Hz}$. Such behavior of the bolometer noise in turn is possibly related to the existence of another noise mechanism with a predominant relaxation in the phase transition region. Considering the experimental data of Figs. 3 and 4 one can conclude that the NEP of the bolometer operating in the transition region improves by a factor of 15 without taking into consideration the effect of hysteresis.

The operation of a $\mathrm{VO}_{2}$-based bolometer in the hysteretic phase transition region is complex due to nonlinear effects and its thermodynamic interaction with the heat sink and environment. The nonlinear effects include memory phenomena, minor loop accommodation and stabilization effects in response to repetitive incident radiation. The overall agreement between the experimental and simulation results validate the proposed mathematical formulation to describe $\mathrm{VO}_{2}$ bolometer operation. For single pulse incident radiation, the responsivity may increase, at least, by a factor of 2 , and for high quality films, the improvement in responsivity may increase by a factor of 6 . The increased single pulse responsivity explains the motivation for employing ac biased bolometer operation to obtain higher responsivity as recently proposed in Ref. 3.

${ }^{1}$ M. Danerud, D. Winkler, M. Lindgren, M. Zorin, V. Trifonov, B. S. Karasik, G. N. Goltsman, and E. M. Gershenzon, J. Appl. Phys. 76, 1902 (1994).

${ }^{2}$ D. P. Osterman, R. Patt, R. Hunt, and J. B. Peterson, Appl. Phys. Lett. 71, 2361 (1997).

${ }^{3}$ C. Reintsema, E. Grossman, and J. Koch, Proc. SPIE 3698, 190 (1999).

${ }^{4}$ C. Chen, X. Yi, X. Zhao, and B. Xiong, 25th International Conference on Infrared and Millimeter Waves, 2000, pp. 211 and 212.

${ }^{5}$ V. Zerov, Y. Kulikov, V. Leonov, V. Malyarov, I. Khrebtov, and I. Shaganov, J. Opt. Technol. 66, 387 (1999).

${ }^{6}$ L. de Almeida, G. S. Deep, A. M. N. Lima, and H. Neff, Appl. Phys. Lett. 77, 4365 (2000).

${ }^{7}$ L. de Almeida, G. S. Deep, A. M. N. Lima, and H. Neff, Opt. Eng. (Bellingham) 41, 2582(2002).

${ }^{8}$ L. de Almeida, G. S. Deep, A. M. N. Lima, and H. Neff, IEEE Trans. Magn. 39, 523 (2003).

${ }^{9}$ G. B. Brandão, L. de Almeida, G. S. Deep, A. M. N. Lima, and H. Neff, J. Appl. Phys. 90, 1999 (2001).

${ }^{10}$ A. M. N. Lima, G. S. Deep, L. de Almeida, H. Neff, and M. Fontana, Conf. Rec. IMTC 2, 919 (2001).

${ }^{11}$ S. Verghese, P. L. Richards, S. A. Sachtjen, and K. Char, J. Appl. Phys. 74, 4251 (1993).

${ }^{12}$ V. Andreev, B. Zakharchenya, Y. Kapshin, V. Noskin, and F. Chudnovskiy, Sov. Phys. JETP 52, 684 (1980).

${ }^{13}$ V. Zerov, Y. Kulikov, V. Malyarov, I. Khrebtov, I. Shaganov, and E. Shadrin, Tech. Phys. Lett. 27, 378 (2001). 